\title{
Magnetic Microstructures From Magnetic Force Microscopy And Monte Carlo Simulation In CoFe-Ag-Cu Granular Films
}

\author{
V.Franco, X.Batlle, A.Valencia, A.Labarta, K.O'Grady and M.L.Watson ${ }^{\text {b }}$ \\ Dept.Física Fundamental, Universidad de Barcelona \\ Diagonal 64708028 Barcelona, Spain \\ ${ }^{\text {a }}$ SEES, University of Wales, Bangor, LL571UT, UK. ${ }^{\text {b } C D S M, ~ C o v e n t r y ~ U n i v e r s i t y, ~ C o v e n t r y ~ U K ~}$
}

\begin{abstract}
CoFe-Ag-Cu granular films, prepared by rf sputtering, displayed magnetic domain microstructures for ferromagnetic concentrations above about $32 \%$ at. and below the percolation threshold. All samples have a fcc structure with an $\langle 111\rangle$ texture perpendicular to the film plane. Magnetic force microscopy (MFM) showed a variety of magnetic domain microstructures, extremely sensitive to the magnetic history of the sample, which arise from the balance of the ferromagnetic exchange, the dipolar interactions and perpendicular magnetocrystalline anisotropy. MFM images indicate that in virgin samples, magnetic bubble domains with an out-of-plane component of the magnetization are surrounded by a quasicontinuous background of opposite magnetization domains. The application of a magnetic field in different geometries drastically modifies the microstructure of the system in the remanent state: i) for an in-plane field, the MFM images show that most of the magnetic moments are aligned along the film plane, ii) for an out-of-plane field, the MFM signal increases about one order of magnitude, and out-of-plane striped domains with alternating up and down magnetization are stabilized. Numerical simulations show that a variety of metastable domain structures (similar to those observed experimentally) can be reached, depending on magnetic history, in systems with competing perpendicular anisotropy, exchange and dipolar interactions.
\end{abstract}

\section{INTRODUCTION}

Nanoscale structures have generated continuous interest in recent years owing to the scientific and technological challenges involved. Among this are granular alloys, which consist of a non-magnetic metallic matrix where nanoferromagnetic (FM) particles are segregated, and which display giant magnetoresistivity (GMR) [1]. This effect is mainly due to the spin-dependent scattering between the conduction electrons and the magnetic moments of the particles [2]. In these systems, for high FM contents but still below the volume percolation threshold, the magnetic interactions between FM entities stabilize a domain structure which affects the transport properties [3]. In particular, structures of striped domains having a net out-of-plane component of the magnetization arranged in an "up" and "down" manner are observed in thin films of granular alloys with a perpendicular component of magnetocrystalline anisotropy [4]. These striped domains are similar to those previously reported in epitaxial hop cobalt films [5], in which the formation of these structures had been explained in terms of the competition between domain-wall and shape energy [6]. However, it is doubtful whether that simple model could be applied to heterogeneous medium such as a granular alloy, in which three phases may be present: pure FM particles, pure non-magnetic particles of matrix component and a metastable FM-matrix alloy, which may be also ferromagnetic. Consequently, such domains could be due to: long range magnetic correlations among FM particles, ferromagnetic behavior in the alloy matrix or a complex interplay between these two mechanisms.

One important question related to striped domains in granular alloys is how the electron scattering in the domain walls affects transport properties. This mechanism is distinct from the spin-dependent scattering, and both may contribute simultaneously to magnetoresistivity effects. Therefore, the resistance of the system may strongly depend on the magnetic history, due to the presence of domain configurations with similar total energy, corresponding to very different geometries of the domain walls. This phenomenon has already been observed in homogeneous Co films [7 ].

In this paper we present a study of the magnetic microstructures of CoFe-Ag-Cu granular alloys, using magnetic force microscopy (MFM). The goal is to characterize the magnetic domains observed for some FM concentrations in this system, and to discuss their stability and morphology after different magnetic processes. It is found that for FM concentrations greater than $40 \%$ at and still below the percolation threshold, which is ca. $60 \%$ at. (50\% in volume) for these samples, the competition between magnetic anisotropy and interparticle interactions stabilizes complex magnetic microstructures that are extremely sensitive to the magnetic history of the sample [3]. Although several samples with different concentrations have been prepared, we only present the results for the sample $\mathrm{Co}_{34} \mathrm{Fe}_{8} \mathrm{Ag}_{54} \mathrm{Cu}_{4}$ because it shows a great variety of magnetic microdomain structures which are fully representative of the different situations observed in this system.

\section{EXPERIMENTAL}

CoFe-Ag-Cu thin films 200-300 nm thick were rf sputtered onto glass substrates at an Ar pressure of 8 mTorr. $25 \%$ of the cobalt content in the classical GMR Co-Ag system was replaced by iron to increase magnetic moment. A small amount of copper was included to improve the natural Ag-Co inmiscibility. The microstructure was determined by: X-ray Diffraction (XRD), Transmission Electron Microscopy (TEM) and Atomic Force Microscopy (AFM). XRD was 
used to determine the average crystalline structure and particles size $(\Theta / 2 \Theta$ scan), and the texture (rocking curves and pole figures).TEM and AFM were used to study the particle size distribution. Selected Area Electron Diffraction (SAED) was used to determine the local crystalline symmetry.

MFM was used to study the domain structure at zero applied field after different magnetic histories (remanent state) and at room temperature.

\section{STRUCTURE}

A detailed study of the microstructure is to be published elsewhere. In this paper we only present the main conclusions. All the samples are granular alloys consisting of a Ag rich matrix, where $\mathrm{CoFe}$ particles are segregated. $\mathrm{Co}$ and $\mathrm{Fe}$ form a solid solution. The degree of crystallinity is low but the homogeneity is high in the whole sample. Ag and CoFe crystals have fcc structure textured with the $\langle 111\rangle$ direction perpendicular to the film plane. The CoFe particles are about $3 \mathrm{~nm}$ in diameter and the mean separation between them is about 1 nm. As shown in XRD and SAED the Ag crystals contain a small amount of $\mathrm{CoFe}$ and vice versa, which changes the crystalline spacing and shifts the position of the XRD peak spectra and the SAED rings. Due to the mixing of the Ag and the $\mathrm{CoFe}$, and plastic and elastic strains produced during deposition, the textured cubes are slightly compressed along the $\langle 111\rangle$ direction, and the magnetocrystalline anisotropy is fairly uniaxial [8].

\section{RESULTS}

This section is devoted to the study of the reorientation of the magnetization from in-plane to out-of-plane directions by MFM imaging and numerical simulation, as a function of the magnetic history. Such reorientation of the magnetization is accompanied by marked changes in the morphology and the arrangement of the magnetic domains. The different configurations observed are constituted by: large in-plane $(\sim 1 \mu \mathrm{m})$ ferrromagnetic domains, perpendicular up-and-down striped domains, and an intermediate state consisting of a distribution of spherical-like magnetic bubbles. MFM images for the ascast $\mathrm{Co}_{34} \mathrm{Fe}_{8} \mathrm{Ag}_{54} \mathrm{Cu}_{4}$ sample after different magnetic treatments are shown in Fig.1.

Fig.1(a) was obtained as deposited, and shows a bubble domain structure, which consists of a network of pseudospherical domains (typical size of $200 \mathrm{~nm}$ ) of the same magnetic polarity surrounded by a quasi-continuous background of opposite magnetization. Such a bubble structure is stable at zero field because repulsion between bubbles of the same magnetization avoids coalescence. The smooth transition between black and white regions (gray areas) corresponds to large reorientation domain walls. Although the out-of-plane magnetic structure seems to be stable in a large scale of time, the recorded MFM signal is weak, indicating that magnetic moments lie almost in plane. When a perpendicular magnetizing field is applied to the former structure and then removed, the out-of-plane component of the magnetization increases significantly and bubbles tend to be more elongated Moreover, the contrast between up and down domains
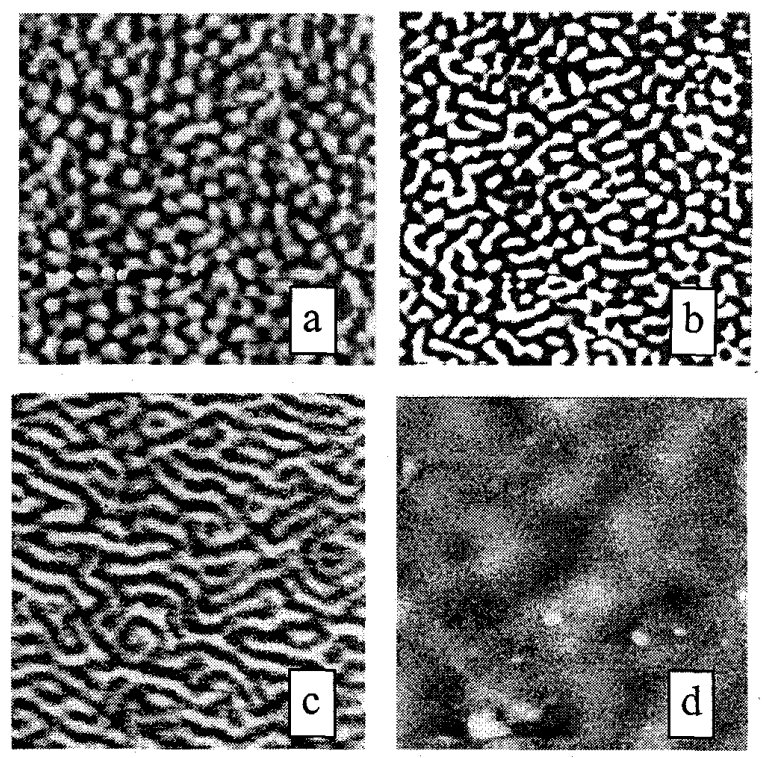

Fig.1. Zero-field MFM images $5 \mu \mathrm{m} \times 5 \mu \mathrm{m}$ of a $\mathrm{Co}_{34} \mathrm{Fe}_{8} \mathrm{Ag}_{54} \mathrm{Cu}_{4}$ thin film. (a) Obtained before any magnetic history, (b) the remanent state obtained after applying $1 \mathrm{~T}$ in the perpendicular direction, (c) after perpendicular demagnetizing cycles were performed and (d) the remanent state after applying an in-plane magnetic field (1 T) to a sample with the bubble domain structure shown in Fig.1(a).

increases which suggests that the magnetization is almost perpendicular in the core of the domains and that the domain walls become narrower. An example of this is shown in Fig.1(b). Elongated bubbles with mean width of $120 \mathrm{~nm}$ corresponding to an MFM signal ten times stronger than that of Fig.1(a), are observed. Because the magnetic field has been applied perpendicular to the film plane, an extra degree of freedom exists and the in-plane orientation of the elongated bubbles is random. After perpendicular demagnetizing cycles were performed, the bubbles coalesced due to wall motion, and labyrinthine stripe domains with alternating up and down magnetization were formed, as shown in Fig.1(c). A certain degree of preferential orientation is observed in this picture, caused by a small in-plane component of the demagnetizing field. The in-plane component of the field tends to align the stripe domains in the field direction, which minimizes the Zeeman energy. In addition, it decreases the outof-plane component of the magnetization and hence a strong enough in-plane field can erase the perpendicular domain structure. Thus, the in-plane demagnetizing process leads to a completely parallel striped domain structure along the direction of the applied field and decreases the MFM signal considerably(not shown).

Fig.1(d) illustrates the remanent state after applying an inplane magnetic field ( $1 \mathrm{~T}$ ) to a virgin sample. As a consequence of this process the MFM signal becomes very weak and large in-plane shapeless ferromagnetic domains $(\sim 1 \mu \mathrm{m})$ are formed. This structure seems to be metastable and slowly evolves towards the original bubble structure (ca. 1 


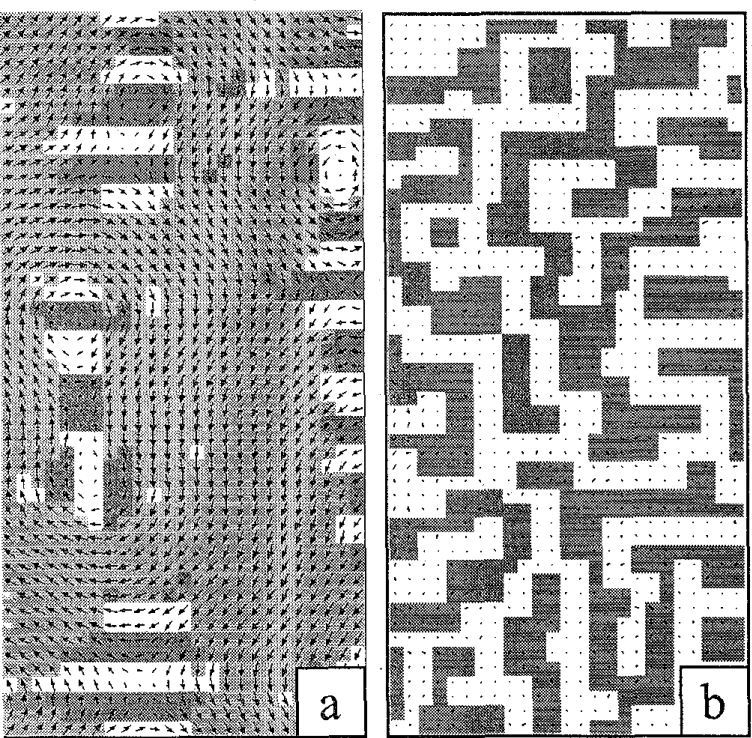

Fig. 2. Detail of the configurations obtained by numerical simulation for the following values of the strength parameters corresponding to anisotropy, ferromagnetic and dipolar interaction : 1.0, 0.38 and 0.21 (in arbitrary reduced units), respectively. Arrows indicate the in-plane component of the spin-direction. Background colors are as follows: white corresponds to perpendicular component of the spins greater than 0.5 , dark gray to perpendicular components smaller than -0.5 and light gray between the formers. (a) Obtained configuration after the simulated annealing. (b) Obtained configuration after the relaxation process.

month at room temperature). Similar results were obtained by applying sufficiently larger in-plane magnetic fields to any of the out- of-plane domain structures observed in these samples. Besides these experimental results, a simplified model was numerically simulated in an attempt to reproduce some of the observed structures. The model consist of a twodimensional square lattice $(50 \times 50)$ of classical spin-one moments which interact through a Heisenberg Hamiltonian and dipolar interactions. A coherent perpendicular anisotropy also exists. By means of the simulated annealing procedure [10] the states of the system at zero temperature coming from the initial totally demagnetized state are investigated. For some specific set of values of the anisotropy, and exchange and dipolar interaction parameters, similar domain structures to those experimentally observed are found. In Fig.2(a) an example of the obtained configurations is shown, which consists of magnetic bubbles with out-of-plane magnetization, surrounded by large in-plane reorientation domains with a vortex-like structure. Inside the bubbles a precursor striped structure is formed (no observed experimentally) because no preferential up and down orientation exists (no field has been applied). In contrast, a very different configuration is reached when the system relaxes following a Monte Carlo simulation, coming from the totally perpendicular magnetized state at zero temperature (Fig.2(b)). In this case, an up and down structure of striped domains with extremely narrow domain walls is stabilized, and the in-plane component of the magnetization is almost zero. The total energy of this configura- tion is very close to that corresponding to the former, suggesting that the ground state of this model has a high degree of degeneration. These preliminary results indicate that a great variety of metastable micro-domain structures can be stabilized depending on the magnetic history, in systems with competing perpendicular anisotropy, exchange and dipolar interactions.

\section{CONCLUSION}

Here we report magnetic domains in granular $\mathrm{CoFe}-\mathrm{Ag}$ $\mathrm{Cu}$ system well below the percolation threshold. These domains are much larger than the mean size of the CoFe particles, suggesting that they are stabilized through interactions among particles which may be propagated by a metastable $\mathrm{CoFe}-\mathrm{Ag}$ alloy matrix. A great variety of domain structures that are highly sensitive to the magnetic history, similar to those found in epitaxial hcp Co films, are observed. Nevertheless, in such granular alloys, magnetic structure can be changed from an in-plane to an out-of-plane configuration following the appropriate magnetic process, without modifying the thickness of the sample. The striped domain structure is assumed to be the most stable one in magnetic media with strong perpendicular anisotropy at the remanent state [9]. However, experimental and numerical results may indicate that in systems with competing perpendicular anisotropy and dipolar and exchange interactions, the striped and the bubble domain structures have similar energy, since both show long stability at the remanent state.

\section{REFERENCES}

[1] A. E. Berkowitz, J. R. Michell, M. J. Carey, A. P. Young, S. Zhang, F. E. Spada, F. T. Parker, H. Hutten and G. Thomas. Phys. Rev. Lett. vol. 68, pp. 5320-5325, May 1992; J. Q. Xiao, J. S. Jiang and C. Chien. ibid., vol. 68 pp. $3749-3753$, June 1992

[2] R. E. Camley and J. Barnas. Phys. Rev. Lett. vol. 63, pp: 664-667, January 1989

[3] X. Batlle, V. Franco, A. Labarta, M. L. Watson and K. O'Grady. Appl. Phys. Lett. vol. 70, pp.132-134, January 1997; V. Franco, X. Batlle, A. Labarta, M. L. Watson and K. O'Grady. J. Appl. Phys. vol. 81 pp. 1-6, April 1997.

[4] A. Gavrin, M. H. Kelley, J. Q. Xiao and C. L. Chien. Appl.Phys.Lett. vol. 66 (13) pp.1683-1685, March 1995; R. Allenspach, M. Stampanoni and A. Bischof. Phys.Rev.Lett. vol.65, pp.3344-3346, 1990; M. Hehn, S. Padovani, K. Oudnajela and J. P. Bucher. Phys. Rev. B, vol. 54(5), pp.3428-3433 August 1996; X. Batlle, V. Franco, A. Labarta, M. L. Watson and K. O'Grady. Appl. Phys. Lett. vol. 70, pp.132-134, January 1997; V. Franco, X. Batlle, A. Labarta, M. L. Watson and K. O'Grady. J. Appl: Phys. vol. 81 pp. 1-6, April 1997.

[5] D. M. Donnet, Kannan M. Krishnan and Y. Yajima. J. Phys. D: Appl. Phys. vol. 28, pp.1942-1950, 1995; M. Hehn, S. Padovani, K. Oudnajela and J. P. Bucher. Phys. Rev. B, vol. 54(5), pp.3428-3433, August 1996.

[6] C. Kittel, Phys. Rev. vol. 70, pp.965, 1946

[7] J. F. Gregg, W. Allen, K. Ounadjela, M. Viret, M. Hehn, S. M. Thompson and J. M. D. Coey. Phys. Rev.Lett., vol. 77(8), pp.1580-1583, August 1996

[8] C. H. Lee, Hui He, F. J. Lamelas, W. Vavra, C. Uher and Roy Clarke. Phys.Rev.B. vol. 42(1), pp.1066-1069, July 1990

[9] S. Kirkpatrick, C. D. Gelatt and M. P.Vecchi. Science vol. 220 (4598), pp.671, 1983.

[10] J. A. Cape and G. W. Lehman. J.Appl.Phys. vol. 42, pp.5732-5735, 1971

e-mail: vfranco at hermes.ffn.ub.es 